\title{
How to translate results from auctions to procurements
}

\author{
Luciano I. de Castro ${ }^{a}$, María-Angeles de Frutos ${ }^{\mathrm{b}, *}$ \\ a Department of Economics, University of Illinois at Urbana-Champaign, United States \\ ${ }^{\mathrm{b}}$ Department of Economics, Universidad Carlos III de Madrid. C/ Madrid, 126. 28903 Getafe, Spain
}

\begin{abstract}
A B S T R A C T
The translation of statements from auctions to procurements is not always straightforward. We define a duality relationship between them and provide the appropriate transformations needed for establishing it. Additionally, we prove that affiliation is preserved under these transformations and establish the linkage principle for procurements.
\end{abstract}

Keywords:

Procurement

Reverse auctions

Linkage principle

Affiliation

JEL classification:

D44

\section{Introduction}

The increasing use of procurements by governments and firms is stimulating research with new and important questions. As a result, more and more economists are studying procurements to understand its properties in order to recommend the best practices. A natural research strategy is to take as a starting point known results in the auction literature. However, as we discuss below, a statement for auctions (where bidders are buyers) is sometimes not readily translatable for procure ments (where bidders are sellers). Since the received literature lacks a simple method to make this translation, researchers in procurement must invest some time in finding the correct parallel statement. The repetition of this effort is obviously inefficient. The objective of this paper is to fill this gap in the literature, by providing a general method to translate the results from one environment to the other.

We motivate the difficulties one can encounter with two examples. First, reverse hazard rate dominance of one distribution over the other suffices to deliver a uniformly more aggressive bidding behavior in an auction. In contrast, to deliver the same result in a procurement one must appeal to hazard rate dominance. Second, if (auction) types are affiliated, available results do not provide grounds for ensuring the affiliation of the dual (procurement) types. It is hence unclear, for instance, the status of the linkage principle for procurements.

We define a duality relationship between auctions and procure ments and provide the appropriate transformations needed for

\footnotetext{
* Corresponding author. Tel.: +34 91 6249600; fax: +34 916249329.

E-mail addresses: decastro.luciano@gmail.com (L.I. de Castro), frutos@eco.uc3m.es (M.-A. de Frutos).
}

establishing it. The Duality Theorem then proves that an equilibrium in one environment corresponds to an equilibrium in the other en vironment. We conclude by illustrating with two applications how to apply the duality result. Those two applications focus on the aforementioned examples. The first one relates to comparative statics on the distribution of types under the private values model. The second one deals with the translation to procurements of the linkage principle under the general affiliated model. To obtain this result, we also generalize a theorem of Milgrom and Weber (1982) that may be of importance by its own.

Finally, the importance of the Duality Theorem goes beyond auction theory. Note that oligopolistic price competition under asymmetric information is formally a variable quantity procurement in which the lowest bidder wins the market and supplies the demanded output at the winning price (see Spulber, 1995). One can hence appeal to the Duality Theorem to get insights on oligopolistic competition models from the auction competition models and vice versa.

\section{Translation of procurements into auctions}

Consider an auction game and a procurement game between $n$ players competing for one object. Assume, for simplicity, that both processes are conducted under the sealed bid format, ${ }^{1}$ and that they are standard in the sense that the best bid or offer determines the winner. Our aim here is to show the duality between the two games. To do so, we first describe the information and the payoff structure in

${ }^{1}$ Both the definitions that follow and the main result can be easily adapted to the open format. 
an auction environment, and then define transformations between the auction and the procurement. The dual of $O$ will be denoted $O^{*}$ with $0^{* *}=0$.

\subsection{Information structure}

Let $(T, \Upsilon, \tau)$ denote the probabilistic space of types in an auction, where $T=\prod_{i}^{n} \quad{ }_{1} T_{i}$. For simplicity, let us assume that each $T_{i}$ is a compact interval, that is, $T_{i}=\left[t_{i}, t_{i}\right] \subset \mathbb{R}_{+}$. The vector of all types $t=\left(t_{i}, t_{-i}\right)$ is distributed according to a non atomic measure $\tau$ over $\prod_{i}^{n}{ }_{1}\left[t_{i}, t_{i}\right]$.

Let $h_{i}: T_{i} \rightarrow T_{i}$ be a bijective function defined by $h_{i}\left(t_{i}\right)=t_{i}+t_{i}-t_{i}$. Note that $h_{\mathrm{i}}^{-1}=h_{i}$. Let $h: T \rightarrow T$ be given by $h(t)=\left(h_{1}\left(t_{1}\right), \ldots, h_{n}\left(t_{n}\right)\right)$. The dual space of types is $T^{*}, \Upsilon^{*}, \tau^{*}$, where $\left(T^{*}, \Upsilon^{*}\right)=(T, Y)$ and $\tau^{*}(A)=$ $\tau\left(h^{-1}(A)\right)$, with the caveat that the dual of type $t_{i} \in\left[t_{i}, t_{i}\right]$ is type

$t_{i}^{*} \equiv h_{i}\left(t_{i}\right)=\bar{t}_{i}+\underline{t}_{i}-t_{i}$.

\subsection{Payoff structure}

An auction payoff structure is a quadruplet $\left(v_{i}, w_{i}, l_{i}, U_{i}\right)_{i}^{n} \quad 1$, where for each buyer $i, v_{i}(t)$ represents the value she assigns to the object, $w_{i}: B^{n} \rightarrow \mathbb{R}$ her payment when the profile of bids is $\mathbf{b}=\left(b, b_{-i}\right) \in B^{n}$ and she wins, $l_{i}: B^{n} \rightarrow \mathbb{R}$ the payment when losing, and $U_{i}: \mathbb{R} \rightarrow \mathbb{R}$ represents her utility. Thus, if buyers $j \neq i$ follow strategies $\beta_{j}: T_{j} \rightarrow B$, buyer $i$ has type $t_{i}$ and bids $b_{i}$ above the reservation price $r$, then her interim expected payoff will be

$$
\begin{aligned}
& \int\left\{U_{i}\left(v_{i}\left(t_{i}, t_{i}\right)-w_{i}\left(b, \beta_{i}\left(t_{i}\right)\right)\right) 1_{\left[b>\max _{j \neq i} \beta_{j}\left(t_{j}\right)\right]}\right. \\
& \left.U_{i}\left(-l_{i}\left(b, \beta{ }_{i}\left(t_{i}\right)\right)\right) 1_{\left[b<\max _{j \neq i} \beta_{j}\left(t_{j}\right)\right]}\right\} \tau\left(d t{ }_{i} \mid t_{i}\right),
\end{aligned}
$$

where $\tau\left(\cdot \mid t_{i}\right)$ is the conditional measure given $t_{i \cdot}{ }^{2}$

Denoted by $\mathrm{v}$ the highest value among bidders, $\mathrm{v} \equiv \max _{i} \sup _{t} v_{i}(t)$. Let the set of bids be $B=[r, v] \subset \mathbb{R}_{+}$. For a given bid $b \in B$, the dual bid is

$b^{*} \equiv \bar{v}+r-b$.

Note that $B^{*}=B$.

Fix an auction payoff structure. Its dual (procurement) payoff structure is $\left(v_{i}^{*}, w_{i}^{*}, l_{i}^{*}, U_{i}^{*}\right)_{i}^{\mathrm{n}} \quad{ }_{1}$, with $^{3}$

$v_{i}^{*}\left(t_{i}^{*}, t^{*}{ }_{i}\right)=\bar{v}+r-v_{i}\left(t_{i}, t_{i}\right)$;

$w_{i}^{*}\left(b_{i}^{*}, b_{i}^{*}\right)=\bar{v}+r-w_{i}\left(b_{i}, b_{i}\right)$;

$l_{i}^{*}\left(b_{i}^{*}, b^{*}{ }_{i}\right)=-l_{i}\left(b_{i}, b_{i}\right)$

$U_{i}^{*}=U_{i}$,

where $t_{j}=t_{j}^{* *}=\left(t_{j}^{*}\right)^{*}$ and $b_{j}=b_{j}^{* *}=\left(b_{j}^{*}\right)^{*}$ for each $j$.

If sellers $j \neq i$ follow strategies $\beta_{j}^{*}: T_{j}^{*} \rightarrow B$ and seller $i$ offers $b^{*}$ below $r$ then her interim expected payoff will be

$$
\begin{aligned}
& \int\left\{U_{i}^{*}\left(w_{i}^{*}\left(b^{*}, \beta^{*}{ }_{i}\left(t^{*}{ }_{i}\right)\right)-v_{i}^{*}\left(t_{i}^{*}, t^{*}{ }_{i}\right)\right) 1_{\left[b^{*}<\min _{j \neq i} \beta_{j}^{*}\left(t_{j}^{*}\right)\right]}\right. \\
& \left.U_{i}^{*}\left(l_{i}^{*}\left(b^{*}, \beta^{*}{ }_{i}\left(t^{*}{ }_{i}\right)\right)\right) 1_{\left[b^{*}>\min _{j \neq i} \beta_{j}{ }^{*}\left(t_{j}^{*}\right)\right]}\right\} \tau^{*}\left(d t^{*}{ }_{i} \mid t_{i}^{*}\right),
\end{aligned}
$$

\footnotetext{
${ }^{2}$ We ignore what happens in a tie for simplicity and because ties occur with zero probability in many auction games.

${ }^{3}$ There are other alternative dual expressions. It is sufficient, for instance, that the duality of types and bids satisfy $t_{j}=t_{j}^{* *}=\left(t_{j}^{*}\right)^{*}$ and $b_{j}=b_{j}^{* *}=\left(b_{j}^{*}\right)^{*}$ and that Eqs. (3) and (4) hold for some $L$ in the place of $\mathrm{v}+r$.
}

where $\tau^{*}\left(\cdot \mid t_{i}^{*}\right)$ is the conditional measure given $t_{i}^{*}$ and $\beta_{j}^{*}$ is the dual strategy of $\beta_{j}$. Note that for each $t$ and its dual $t^{*}, \beta_{i}\left(t_{i}\right)>\max _{j \neq i} \beta_{j}\left(t_{j}\right)$ iff $\beta_{i}^{*}\left(t_{i}^{*}\right)>\min _{j \neq i} \beta_{j}^{*}\left(t_{j}^{*}\right)$.

Definition 1. An auction environment is a profile $\mathcal{A}=\left((T, \Upsilon, \tau),\left(v_{i}, w_{i}\right.\right.$, $\left.\left.l_{i}, U_{i}\right)_{i}^{n}{ }_{1}\right)$ formed by the elements described above. Its dual envi ronment is the profile $\mathcal{P}=\mathcal{A}^{*}=\left(\left(T^{*}, \Upsilon^{*}, \tau^{*}\right),\left(v_{i}^{*}, w_{i}^{*}, l_{i}^{*}, U_{i}^{*}\right)_{i}^{n}{ }_{1}\right)$ and it corresponds to a procurement environment.

The following example illustrates this definition:

Example 1. Consider a private values first price auction $\mathcal{A}$ with inde pendent but asymmetric types given by

$$
\begin{aligned}
\tau\left(\left\{t \in \prod_{i}^{n}\left[t_{i}, \bar{t}_{i}\right]: t_{i} \leq x_{i}, \forall i\right\}\right) & =\prod_{i}^{n} G_{i}\left(x_{i}\right) ; \\
v_{i}\left(t_{i}, t_{i}\right) & =t_{i} ; \\
w_{i}\left(b_{i}, b_{i}\right) & =b_{i} ; \\
l_{i}(b) & =0 .
\end{aligned}
$$

Then, the procurement $P$ defined by the expressions below is its dual:

$$
\begin{aligned}
\tau^{*}\left(\left\{t^{*} \in \prod_{i}^{n}\left[\underline{t}_{i}^{*}, \bar{t}_{i}^{*}\right]: t_{i}^{*} \leq x_{i}, \forall i\right\}\right) & =\prod_{i}^{n} F_{i}\left(x_{i}\right), \\
v_{i}^{*}\left(t_{i}^{*}, t^{*}{ }_{i}\right) & =\bar{v}+r-t_{i} ; \\
w_{i}^{*}\left(b_{i}^{*}, b^{*}{ }_{i}\right) & =\bar{v}+r-b_{i} ; \\
l_{i}^{*}\left(b_{i}^{*}, b^{*}{ }_{i}\right) & =0,
\end{aligned}
$$

where $F_{i}\left(x_{i}\right)=1-G_{i}\left(t_{i}+t_{i}-x_{i}\right) ; t_{i}=t_{i}+t_{i}-t_{i}^{*}$, and $b_{i}=v+r-b_{i}^{*}$. Notice that $v_{i}^{*}\left(t_{i}^{*}, t_{-i}^{*}\right)=v+r+t_{i}^{*}-t_{i}-t_{i} \neq t_{i}^{*}$, unless $v+r=t_{i}+t_{i}$.

The following is the heart of our contribution:

Theorem 1 (Duality Theorem). The auction environment $\mathcal{A}=((\mathrm{T}, \Upsilon$, $\left.\tau),\left(v_{i}, w_{i}, l_{i}, U_{i}\right)_{i}^{n}{ }_{1}\right)$ and its dual $\mathcal{P}=\left(\left(\mathrm{T}^{*}, \Upsilon^{*}, \tau^{*}\right),\left(v_{i}^{*}, w_{i}^{*}, l_{i}^{*}, U_{i}^{*}\right)_{i}^{n} \quad 1\right)$ are strategically equivalent games.

More formally, if players in $\mathcal{A}$ follow the strategy profile $\beta=\left(\beta_{1}, \ldots\right.$, $\beta_{n}$ ) and players in $\mathcal{P}$ follow the dual strategy profile $\beta^{*}=\left(\beta_{1}^{*}, \ldots, \beta_{n}^{*}\right)$, then their (ex ante, interim, ex post) payoffs will be exactly the same. Thus, $\beta$ is an equilibrium of $\mathcal{A}$ iff its dual $\beta^{*}$ is an equilibrium of $\mathcal{P}$.

Proof. The ex post payoff of player $i$ in $\mathcal{A}$ is:

$$
\begin{gathered}
U_{i}\left(v_{i}\left(t_{i}, t{ }_{i}\right)-w_{i}\left(\beta_{i}\left(t_{i}\right), \beta_{i}\left(t_{i}\right)\right)\right) 1_{\left[\beta_{i}\left(t_{i}\right)>\max _{j \neq i} \beta_{j}\left(t_{j}\right)\right]} \\
\quad+U_{i}\left(-l_{i}\left(\beta_{i}\left(t_{i}\right), \beta_{i}\left(t_{i}\right)\right)\right) 1_{\left[\beta_{i}\left(t_{i}\right)<\max _{j \neq i} \beta_{j}\left(t_{j}\right)\right]}
\end{gathered}
$$

while in $\mathcal{P}$ is:

$$
\begin{gathered}
U_{i}^{*}\left(w_{i}^{*}\left(\beta_{i}^{*}\left(t_{i}^{*}\right), \beta^{*}{ }_{i}\left(t^{*}{ }_{i}\right)\right)-v_{i}^{*}\left(t_{i}^{*}, t^{*}{ }_{i}\right)\right) 1_{\left[\beta_{i}^{*}\left(t_{i}^{*}\right)<\min _{j \neq i} \beta_{j}^{*}\left(t_{j}^{*}\right)\right]} \\
+U_{i}^{*}\left(l_{i}^{*}\left(\beta_{i}^{*}\left(t_{i}^{*}\right), \beta^{*}{ }_{i}\left(t^{*}\right)\right)\right) 1{ }_{\left[\beta_{i}^{*}\left(t_{i}^{*}\right)>\min _{j \neq i} \beta_{j}^{*}\left(t_{j}^{*}\right)\right] .}
\end{gathered}
$$

Substituting $\beta_{i}^{*}\left(t_{i}^{*}\right)$ by $\bar{v}+r-\beta_{i}\left(t_{i}\right)$, it follows that that the event $\left[\beta_{i}\left(t_{i}\right)>\max _{j \neq i} \beta_{j}\left(t_{j}\right)\right]$ is equal to the event $\left[\beta_{i}^{*}\left(t_{i}^{*}\right)<\min _{j \neq i} \beta_{j}^{*}\left(t_{j}^{*}\right)\right]$. The same holds true for events $\left[\beta_{i}\left(t_{i}\right)<\max _{j \neq i} \beta_{j}\left(t_{j}\right)\right]$ and $\left[\beta_{i}^{*}\left(t_{i}^{*}\right)>\right.$ $\left.\min _{j \neq i} \beta_{j}^{*}\left(t_{j}^{*}\right)\right]$. Furthermore, $U_{i}^{*}\left(l_{i}^{*}\left(\beta_{i}^{*}\left(t_{i}^{*}\right), \beta_{-\mathrm{i}}^{*}\left(t_{-i}^{*}\right)\right)\right)=U_{i}\left(l_{i}\left(\beta_{i}\left(t_{i}\right)\right.\right.$, $\left.\left.\beta-i\left(t_{-i}\right)\right)\right)$ and

$$
\begin{aligned}
& U_{i}\left(v_{i}\left(t_{i}, t_{i}\right)-w_{i}\left(\beta_{i}\left(t_{i}\right), \beta_{i}\left(t_{i}\right)\right)\right) \\
& \quad=U_{i}^{*}\left(\bar{v}+r-v_{i}^{*}\left(t_{i}^{*}, t^{*}{ }_{i}\right)-\bar{v}-r+w_{i}^{*}\left(\beta_{i}^{*}\left(t_{i}^{*}\right), \beta^{*}{ }_{i}\left(t_{i}^{*}\right)\right)\right) \\
& \quad=U_{i}^{*}\left(w_{i}^{*}\left(\beta_{i}^{*}\left(t_{i}^{*}\right), \beta^{*}{ }_{i}\left(t^{*}{ }_{i}\right)\right)-v_{i}^{*}\left(t_{i}^{*}, t^{*}{ }_{i}\right)\right) .
\end{aligned}
$$

Since ex post payoffs are equal, the statement follows. 


\section{Application to stochastic orders}

Existing work on comparative statics for first price auctions has shown that a stochastically higher distribution of values (in the sense of the reverse hazard rate order) leads to uniformly more aggressive bidding (see Lebrun (1998) and Maskin and Riley (2000)). We first show how these results translate to procurement games by appealing to the Duality Theorem.

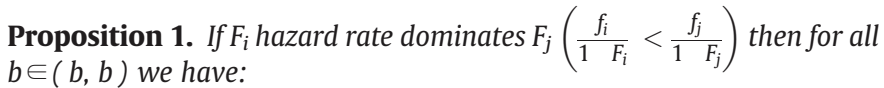

i) $\left(\beta_{i}^{*}\right)^{-1}(b)>\left(\beta_{j}^{*}\right)^{-1}(b)$, and

ii) $F_{i}\left(\left(\beta_{i}^{*}\right)^{-1}(b)\right)<F_{j}\left(\left(\beta_{j}^{*}\right)^{-1}(b)\right)$.

Proof. By Example (1), the dual of the procurement game is an auction with distribution of types $G_{1}, G_{2}$ such that $G_{i}(x) \equiv 1-F_{i}\left(t_{i}-x\right)$. Hazard rate dominance of $F_{i}$ over $F_{j}$ implies reverse hazard rate dominance of $G_{j}$ over $G_{i}$. Thus, Propositions 3.3 and 3.5 in Maskin and Riley (2000) deliver the comparative statics above.

It is worth mentioning that Pesendorfer (2000) shows the above result by analyzing the system of differential equations that determine the equilibrium inverse bidding functions. The current proof illustrates the usefulness of the Duality Theorem.

Proposition 2. Consider a competitive procurement and let $\beta_{1}^{*}, \beta_{2}^{*}$ be the equilibrium bid functions when cost distributions are $\left(F_{1}, F_{2}\right)$ and let $\tilde{\beta}_{1}^{*}, \tilde{\beta}_{2}^{*}$ be the equilibrium bid functions when cost distributions are $\left(F_{1}, \tilde{F}_{2}\right)$ If $\tilde{F}_{2}$ hazard rate dominates $F_{2}$, then for all $b^{*} \in(b, b)$ we have:

i) $\tilde{\beta}_{1}^{*}\left(t^{*}\right)>\beta_{1}^{*}\left(t^{*}\right)$, and

ii) $\tilde{F}_{2}\left(\left(\tilde{\beta}_{2}^{*}\right)^{-1}\left(b^{*}\right)\right)<F_{2}\left(\left(\tilde{\beta}_{2}^{*}\right)^{-1}\left(b^{*}\right)\right)$.

Proof. Theorem 1 in Lebrun (1998) and Theorem 1 deliver the result.

Propositions 1 and 2 show that the conditions on the primitives to have a uniformly more aggressive bidding behavior are different in auctions and in procurements. In the former, reverse hazard rate dominance of one distribution over the other suffices, whereas the latter one must appeal to hazard rate dominance.

More recently, the impact of a less dispersed distribution of types on auction bidding has been analyzed by Hopkins and Kornienko (2007). From their comparative statics results and the Duality Theorem, the effects of more precise information on procurement bidding is next obtained.

Proposition 3. Consider a competitive procurement. If $f_{i} / f_{j}$ is unimodal and $E_{i}[t] \geq E_{j}[t]$, then $\beta_{i}^{*}\left(t^{*}\right)>\beta_{j}^{*}\left(t^{*}\right)$ on $\left(t, \hat{t}_{H}\right]$ where $\hat{t}_{H}$ is the maximum on $[t, t]$ of the probability ratio $\left(1-F_{1}\right) /\left(1-F_{2}\right)$.

Further, if $\hat{t}_{H}<\mathrm{t}$ then $\beta_{i}^{*}\left(t^{*}\right)$ can cross $\beta_{j}^{*}\left(t^{*}\right)$ once and from above on $\left(\hat{t}_{H}, \mathrm{t}\right)$.

\section{Application to the linkage principle and revenue ranking}

The linkage principle is an important result in auction theory, which allows to show that the second price auction yields higher revenue than the first price auction if types are affiliated (see Krishna, 2002). Does it hence follow that the "second price procurement" is preferable over the "first price procurement"? If the second price auction is the one that gives higher payments, an auctioneer might actually prefer the first price procurement as she is the one making the payments in a procurement. It is even reasonable to doubt the validity of the principle itself, as the affiliation inequality for procure ments is the same as for auctions, but the best type is the lowest type rather than the highest type. Therefore, inequalities may not even have the direction necessary to produce a proof. To make things even more dubious, the received literature does not ensure that affiliation is preserved under decreasing transformations, as needed to translate types from auctions to procurement. ${ }^{4}$ In sum, it is not clear at all the status of the linkage principle for procurements. This section clarifies this issue.

We begin by extending Milgrom and Weber (1982)'s Theorem 3 for non increasing transformations, proving that affiliation is pre served under the duality maps.

Theorem 2. Assume that $X_{1}, \ldots, X_{n}$ are affiliated. If $g_{1}, \ldots g_{n}$ are all non decreasing or are all non increasing functions from $\mathbb{R}$ to $\mathbb{R}$ then $g_{1}\left(X_{1}\right)$,... $g_{n}\left(X_{n}\right)$ are affiliated.

Proof. Using Theorem 3 of Milgrom and Weber (1982), it is sufficient to argue that if $X_{1}, \ldots, X_{n}$ are affiliated, then $-X_{1}, \ldots,-X_{n}$ are also affiliated, since $g_{1}, \ldots, g_{n}$ are all non increasing if and only if $g_{1} \circ S, \ldots$, $g_{n} \circ s$ are all non decreasing, where $s(x)=-x$.

In the particular case in which there is a density function $f$ : $\mathbb{R}^{n} \rightarrow \mathbb{R}_{+}$, affiliation is equivalent to $f(\mathrm{x}) f(y) \leqslant f(x \wedge y) f(x \vee y)$. Since $-(x \wedge y)=(-x) \vee(-y)$ and $-(x \vee y)=(-x) \wedge(-y)$, affiliation of $f$ is then equivalent to affiliation of $\hat{\mathrm{f}}: \mathbb{R}^{n} \rightarrow \mathbb{R}_{+}$, where $\hat{\mathrm{f}}(x)=(f \circ s)(x)=$ $f(-x)$, as $f(-x) f(-y) \leqslant f((-x) \wedge(-y)) f((-x) \vee(-y))$.

For the general case, we say that a set $A \subset \mathbb{R}^{n}$ is increasing if its indicator function $1_{A}$ is non decreasing in the usual partial order of $\mathbb{R}^{n}$. A set $S \subset \mathbb{R}^{n}$ is a sublattice if $x \wedge y \in S$ and $x \vee y \in S$ for all $x, y \in S$. For a vector of random variables $X=\left(X_{1}, \ldots, X_{n}\right)$ write $P(A)$ for $\operatorname{Pr}(X \in A)$ and $P(-A)$ for $\operatorname{Pr}(-X \in A)=\operatorname{Pr}(X \in-A)$. $X$ is affiliated iff $P(A B \mid S) \geqslant$ $P(A \mid S) P(B \mid S)$, for all increasing sets $A, B$ and sublattice $S$, where $A^{C}=$ $\mathbb{R}^{n} \backslash A$.

We want to prove that $X$ affiliated implies $-X$ affiliated, that is,

$\operatorname{Pr}(-X \in A \cap B \mid S)=P((-A)(-B) \mid S) \geqslant P(-A \mid S) P(-B \mid S)$,

for all increasing sets $A, B$ and sublattice $S$. Fix $A$ and $B$ increasing sets. Then, $(-A)^{c}$ and $(-B)^{c}$ are also increasing. Thus, $P(-A \mid S)=1-$ $P\left((-A)^{c} \mid S\right)$ and $P(-B / S)=1-P\left((B)^{c} \mid S\right)$, which implies:

$P(-A \mid S) P(-B \mid S)=1-P\left((-A)^{c} \mid S\right)-P\left((-B)^{c} \mid S\right)+P\left((-A)^{c} \mid S\right) P\left((-B)^{c} \mid S\right)$.

Similarly, $\left.P((-A)(-B) \mid S)=1-P\left((-A)^{c} \mid S\right)-P\left((-B)^{c} \mid S\right)\right)+P\left((-A)^{c}\right)$ $\left.(-B)^{c} \mid S\right)$. Thus, $P((-A)(-B) \mid S) \geqslant P(-A \mid S) P(-B \mid S)$, iff

$P\left((-A)^{c} \cap(-B)^{c} \mid S\right) \geqslant P\left((-A)^{c} \mid S\right) P\left((-B)^{c} \mid S\right)$,

but since $(-A)^{c}$ and $(-B)^{c}$ are increasing sets, this last equation is true because $X$ is affiliated.

The reader should note that the above theorem would be false if we request the functions $g_{i}$ to be merely monotonic. ${ }^{5}$

We can now apply the Duality Theorem to obtain easily a linkage principle for procurements:

Theorem 3. Assume that the bidders are symmetric, risk neutral, have affiliated types, and that the same reserve price is used in both a first price and in a second price procurement. If the players play the symmetric equilibrium, then the auctioneer prefers the second price over the first price procurement.

Proof. It is well known that $E\left[w_{I}\left(t_{i}, t_{-i}\right)\right] \leq E\left[w_{I I}\left(t_{i}, t_{-i}\right)\right]$, where $w_{I}\left(t_{i}, t_{-i}\right)$ and $w_{\text {II }}\left(t_{i}, t_{-i}\right)$ denote, respectively, the symmetric payments in the first and in the second price auctions. By Eq. (4), this implies that in the dual procurement, $E\left[w_{\mathrm{I}}^{*}\left(t_{i}, t_{-i}\right)\right] \geq E\left[w_{\mathrm{II}}^{*}\left(t_{i}, t_{-i}\right)\right]$. Since $E\left[w_{P}^{*}\left(t_{i}, t_{-i}\right)\right]$ is the expected payment that the auctioneer has to make in the procurement $P=\mathrm{I}, \mathrm{II}$, then she prefers the second price mechanism.

\footnotetext{
${ }^{4}$ Milgrom and Weber (1982)'s Theorem 3 states that: if $g_{1}, \ldots, g_{n}$ are non-decreasing functions from $\mathbb{R}$ to $\mathbb{R}$ and $X_{1}, \ldots X_{n}$ are affiliated then $g_{1}\left(X_{1}\right) \ldots, g_{n}\left(X_{n}\right)$ are affiliated.

${ }^{5}$ If $g_{1}$ is increasing and $g_{2}$ is decreasing, the inequalities that guarantee that $X_{1}$ and $X_{2}$ are affiliated will be the opposite to the ones guaranteeing that $g\left(X_{1}\right)$ and $g\left(X_{2}\right)$ are.
} 


\section{References}

Hopkins, E., Kornienko, T., 2007. Cross and double cross: comparative statics in first price and all pay auctions. The B. E. Journal of Theoretical Economics 7 Article 19. Krishna, V., 2002. Auction Theory. Academic Press, San Diego.
Lebrun, B., 1998. Comparative statics in first price auctions. Games and Economic Behavior 25, 97-110.

Maskin, E., Riley, J., 2000. Asymmetric auctions. The Review of Economic Studies 67, 413-438.

Milgrom, P., Weber, R., 1982. A theory of auctions and competitive bidding. Econometrica $50,1089-1122$.

Pesendorfer, M., 2000. a study of collusion in first-price auctions. The Review of Economic Studies 67, 381-411.

Spulber, D., 1995. Bertrand competition when rivals' costs are unknown. The Journal of Industrial Economics 43, 1-11. 